\title{
Sexual function, self-esteem, and general well- being in Chinese adult survivors of childhood cancers: a cross-sectional survey
}

\author{
CF Ng *, Cindy YL Hong, Becky SY Lau, Jeremy YC Teoh, Samuel CH Yee, Alex WK Leung, John WM Yuen
}

This article was published on 9 Oct 2019 at www.hkmj.org.

\section{A B S T R A C T}

Introduction: This study was conducted to evaluate sexual function in adult survivors of childhood cancers and investigate possible relationships between sexual function and quality of life, as measured by general well-being, self-esteem, body image, and depressive symptoms.

Methods: This cross-sectional survey was performed in our centre from 14 August 2015 to 8 September 2017. Adult patients who had a history of childhood cancers, and who were disease-free for $>3$ years, were approached for the study during clinical followup. Clinical information was collected from medical records. Self-administered questionnaires regarding quality of life and sexual functioning were given to the patients and resulting data were analysed.

Results: Two hundred patients agreed to participate in the study. The overall response rate was $64.8 \%$. Ninety-one $(45.5 \%)$ patients were women, and the mean age was $25.4 \pm 5.57$ years. The overall sexual functioning score was $28.3 \pm 20.09$. Forty-eight (24.0\%) patients reported at least one sexual problem. Among patients who reported no sexual problems, more had haematological cancers $(\mathrm{P}=0.009)$, fewer underwent surgery $(\mathrm{P}=0.004)$, fewer underwent surgery with external effects $(P=0.032)$, and fewer were regular social drinkers $(\mathrm{P}=0.013)$; additionally, they had a higher mean Rosenberg self-esteem scale score $(\mathrm{P}=0.010)$, lower mean body image scale score $(\mathrm{P}=0.008)$, and lower mean Patient Health Questionnaire score $(\mathrm{P}=0.001)$.

Conclusion: Aspects of life beyond disease condition and physical function should be considered in adult survivors of childhood cancers. Appropriate referral and intervention should be initiated for these patients when necessary.

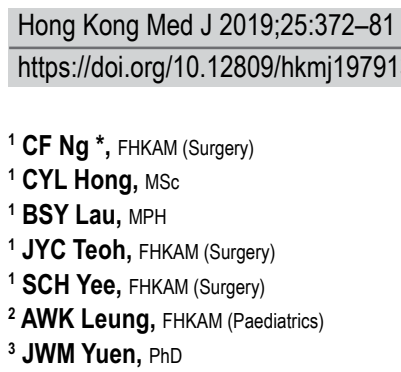

School of Nursing, The Hong Kong Polytechnic University, Hung Hom, Hong Kong

* Corresponding author: ngcf@surgery.cuhk.edu.hk

\section{New knowledge added by this study}

- Approximately one-quarter of young Chinese cancer survivors in Hong Kong had at least one sexual problem.

- Sexual problems were more common in men, in patients diagnosed with cancer at an older age, and in patients who were married or had a history of sexual experiences.

- The presence of sexual problems in adult survivors of childhood cancer was significantly associated with a history of surgery, as well as a history of surgery with external effects; patients with sexual problems generally had lower physical well-being scores, lower self-esteem scores, higher body image scale scores, and an increased number of depressive symptoms.

Implications for clinical practice or policy

- Rather than entirely focusing on disease condition and physical function, physicians and medical staff should ensure that they consider other aspects of life in survivors of childhood cancer, to support holistic recovery of these patients.

- Multidisciplinary care, such as involvement of adult urologists and gynaecologists, would facilitate the transition of these young cancer survivors into adult life.

\section{Introduction}

Sexual health, defined by the World Health Organization as a state of physical, emotional, mental and social well-being related to sexuality, has been recognised as an integral part of overall health and quality of life. ${ }^{1}$ Improvements in disease understanding and treatment options have changed the circumstances involved in the management of sexual dysfunction and reproductive medicine.

With improvements in cancer care, the long-term outlook of paediatric cancer patients has significantly improved in recent decades. ${ }^{2}$ 
However, there is increasing awareness of the problems experienced by these cancer survivors when they reach adulthood. ${ }^{3}$ Potential problems experienced by adult survivors of childhood cancers include (1) physical and functional complications related to the cancer and its therapies (eg, delay in pubertal development, hormonal production, azo-/ oligospermia, ovarian failure, and vaginal stenosis); and (2) psychosocial problems related to the cancer and its therapies (eg, concerns regarding cancer recurrence, self-esteem, and relationship problems). These physical and psychological dysfunctions affect the sexual health and overall health of the patients. The findings of many reports have suggested significant associations between sexual function and health status, ${ }^{4}$ and have revealed that these problems are relatively common among cancer survivors. ${ }^{5,6}$

Unfortunately, discussions of sexual dysfunction remain infrequent in traditional Chinese culture. The situation may be more difficult among young adult cancer survivors. Therefore, information regarding cancer-related sexual dysfunction, including its prevalence in the Chinese population, remains limited and may lead to an underestimation of the seriousness of the problem. To address this lack of knowledge and facilitate future development of childhood cancer care, this cross-sectional study was conducted to evaluate sexual function in adult survivors of childhood cancers and the relationships of sexual function with the general well-being, selfesteem, body image, and depressive symptoms of these patients.

\section{Methods}

\section{Patients}

The study was conducted in accordance with the Declaration of Helsinki and was performed at The Chinese University of Hong Kong. The sample size was based on a convenience sample of all patients that we could recruit during the 2-year study period. Consecutive patients who were returning to the paediatric oncology clinic for follow-up and who fulfilled the inclusion and exclusion criteria were invited to participate in the study. After patients provided informed consent, basic demographic data and disease-related information were collected.

The inclusion criteria were as follows: (1) diagnosed with cancer at age <18 years; (2) aged 18 to 40 years at the time of inclusion in the study; (3) not undergoing treatment and disease-free $>3$ years after completing treatment (excluding use of chemopreventive agents). The exclusion criteria were as follows: (1) original tumours that were hormone-dependent, such as breast cancer; (2) ongoing sex hormone supplementation; and (3) sensory/cognitive impairment that would interfere with the patient's ability to independently complete the questionnaires.

\section{華裔兒時癌症成年生存者的性功能、自尊及 整體福祉：橫斷面研究}

吳志輝、匡若琳、劉瑞欣、張源津、余知行、梁永妿、袁偉文

引言: 本研究旨在評估兒時癌症成年生存者的性功能, 以及其性功能

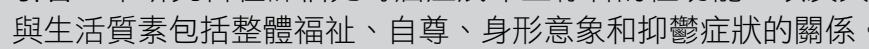

方法：此橫斷面研究於 2015 年 8 月 14 日至 2017 年9月 8 日進行。於覆 診時, 曾患有兒時癌症及康復 3 年以上的成年人會被詢問參與研究意 向。臨床資料於醫料記錄中提取及派發有關生活質素及性功能的問卷 予病人自行填寫而其後作數據分析。

結果：200名病人同意參與研究。整體回應率為 $64.8 \%$ 。91名 ( $45.5 \%)$ 為女性, 平均年齡為 $25.4 \pm 5.57$ 歲。整體平均性功能分數為 $28.3 \pm 20.09 \circ 48$ 名 $(24.0 \%)$ 病人表示患有至少一項性功能問題。在 表示沒有性功能問題的病人中, 較多人曾有血液癌症 $(\mathrm{P}=0.009)$

較少人曾進行影響外觀的手術 $(\mathrm{P}=0.032)$ 及較少慣性社交酒民

$(\mathrm{P}=0.013)$, 同時他們有較高的羅森伯格（Rosenberg）自尊心分數

$(\mathrm{P}=0.010)$ 、較低身形意象分數（P=0.008）及患者健康問卷分數 $(\mathrm{P}=0.001)$

結論：應考慮兒時癌症成年生存者疾病及身體功能以外的生活問題。 可主動提供適當的轉介及治療予有需要的病人。

\section{Questionnaire data collection}

A series of self-administered questionnaires were completed by the patients in a private room in the clinic. The following questionnaires were used.

\section{Medical outcomes study sexual functioning scale}

This is a validated instrument that has been widely used to identify sexual impairment and dysfunction associated with serious health conditions or sideeffects of treatments. ${ }^{7}$ It consists of four questions for both male and female patients, which evaluate sexual problems including lack of interest in sexual activity, difficulty in becoming aroused, difficulty in relaxing and enjoying sex, and difficulty in achieving orgasm. Each outcome is measured with an ordinal scale ranging from 0 ('not a problem') to 4 ('very much a problem'). The category of 'not applicable' was recoded as 0 during calculation. Total scores were calculated and transformed to a 0-100 scale; a higher score indicates more sexual problems. The questionnaire was translated and validated by Department of Nursing, The Polytechnic University of Hong Kong.

\section{General Health Questionnaire Short Form-12}

This questionnaire is used for general measurement of health status in terms of physical component score (PCS) and mental component score (MCS). ${ }^{8}$ The summary scores are calculated based on the standard scoring algorithm described in the manual ${ }^{9}$; a higher score represents better physical or mental health. 


\section{Rosenberg self-esteem scale}

This tool is commonly used to evaluate selfesteem. ${ }^{10,11}$ It comprises 10 questions which assess both positive and negative feelings about the self. Patients respond to questions using a 4-point scale, ranging from 'strongly agree' to 'strongly disagree'; a higher summary score indicates higher self-esteem.

\section{Body image scale}

Body image scale (BIS) is an 11-item scale used to assess body image changes in cancer survivors after cancer treatment. ${ }^{12,13}$ Body image changes can be rated in an ordinal scale ranging from 0 ('not at all') to 3 ('very much'); a higher total score indicates greater body image distress.

\section{Patient Health Questionnaire}

Patient Health Questionnaire (PHQ-9) is widely used to measure depressive symptoms. It assesses the extent to which the symptoms were experienced by the patient in past 2 weeks. ${ }^{14,15}$ Patients respond to items using an ordinal scale ranging from 0 ('not at all') to 3 ('nearly every day'). The degree of depression is graded based on the total score of the nine items: mild ( $5 \leq$ score $\leq 9)$, moderate $(10 \leq$ score $\leq 14)$, and severe (score $\geq 15$ ).

\section{Statistical analysis}

Descriptive statistics are presented as counts and percentages for categorical data, and as means and standard deviations for continuous data. The Chi squared test, Fisher's exact test, analysis of variance, correlation, and simple linear regression methods were used for simple analyses and subgroup comparisons. More sophisticated analyses were performed using multiple linear regression and multivariable logistic regression, to control for potential confounders. All statistical analyses were performed using SPSS (Windows version 24.0; IBM Corp, Armonk [NY], United States). All levels of significance were set at the 0.05 level and all tests were two-sided. Missing data were excluded from analysis.

\section{Results}

\section{Patient demographic characteristics and cancer treatment histories}

The study was performed from 14 August 2015 to 8 September 2017. Three hundred seventy-two consecutive patients were approached, and 241 patients agreed to participate in the study. The overall response rate was $64.8 \%$. Forty-one patients were excluded from analysis due to incomplete data collection or failure to appropriately meet the inclusion/exclusion criteria. Therefore, a total of 200 patients were included in the analysis.
Among the 200 patients, 91 (45.5\%) were women; the mean age of all patients was $25.4 \pm$ 5.57 years, and the mean age at diagnosis was 7.8 \pm 5.09 years. In total, $133(66.5 \%)$ patients had haematological cancer, among whom 92 (46.0\%) had acute lymphoid leukaemia, 15 (7.5\%) had acute myeloid leukaemia, and $10(5.0 \%)$ had Hodgkin lymphoma. Sixty-seven (33.5\%) patients had nonhaematological cancer, among whom 11 (5.5\%) had Wilm's tumour, $10(5.0 \%)$ had osteosarcoma, and eight (4.0\%) had neuroblastoma. Sixty-five (32.5\%) patients underwent surgery, and 23 (11.5\%) exhibited visible external effects, such as limb resection. Fiftythree $(26.8 \%)$ patients received radiotherapy and 176 (88.9\%) received chemotherapy. Ten $(5.0 \%)$ patients had experienced cancer relapse. Thirty-one (15.5\%) patients were married, 94 (47.0\%) were single with a current or previous relationship, and 75 (37.5\%) were single and had never been in a relationship (Table 1). Eighty (40.4\%) patients reported a history of sexual experiences and 15 (7.6\%) had impregnated their partners or ever conceived a child.

\section{Sexual impairment and dysfunction related to childhood cancer and treatment}

The overall medical outcomes study sexual functioning score was $28.3 \pm 20.09$. Men (32.3 \pm 19.92 ) had a significantly higher mean sexual functioning score (ie, more sexual problems) than women $(23.6 \pm 19.36, \mathrm{P}=0.002)$ [Table 2]. Age at the time of this study $(r=0.193, \mathrm{P}=0.006)$ and age at cancer diagnosis $(r=0.147, \mathrm{P}=0.037)$ were significantly positively correlated with sexual functioning score (ie, more sexual problems). Patients who had a history of sexual experiences $(32.8 \pm 19.45, \mathrm{P}=0.008)$ or who had been married $(38.1 \pm 20.63, \mathrm{P}=0.004)$ had a significantly higher mean sexual functioning score than patients who had no history of sexual experiences and who had not been married (Table 2). Multiple regression analysis controlling for all potential confounders suggested that male sex $(\beta=9.20, P=0.001)$ and marital status of 'married' $(\beta=13.95, P=0.038)$ were significantly associated with higher sexual functioning score (ie, more sexual problems) [Table 3].

\section{Assessments of self-esteem, body image, and depression in all patients}

The mean Rosenberg self-esteem scale score was $29.9 \pm 4.25$. Multiple regression analysis showed that a history of relapse $(\beta=-2.89, \mathrm{P}=0.044)$ was significantly associated with Rosenberg self-esteem scale score following adjustment for other variables (ie, patients who had a history of relapse had lower self-esteem) [online Supplementary Appendices 1 and 2].

The mean BIS score was $5.6 \pm 4.45$. Age at diagnosis was statistically significantly positively 
TABLE I. Demographic data of the entire cohort $(n=200)^{*}$

\begin{tabular}{|c|c|}
\hline Demographics & Value \\
\hline Age (years) & $25.4 \pm 5.57$ \\
\hline \multicolumn{2}{|l|}{ Sex } \\
\hline Female & $91(45.5 \%)$ \\
\hline Male & $109(54.5 \%)$ \\
\hline Body mass index $\left(\mathrm{n}=198, \mathrm{~kg} / \mathrm{m}^{2}\right)$ & $22.3 \pm 3.59$ \\
\hline \multicolumn{2}{|l|}{ Cancer type } \\
\hline Haematological cancer & $133(66.5 \%)$ \\
\hline Acute lymphoid leukaemia & $92(69.2 \%)$ \\
\hline Acute myeloid leukaemia & $15(11.3 \%)$ \\
\hline Hodgkin lymphoma & $10(7.5 \%)$ \\
\hline Other & $16(12.0 \%)$ \\
\hline Non-haematological cancer & $67(33.5 \%)$ \\
\hline Wilm's tumour & $11(16.4 \%)$ \\
\hline Osteosarcoma & $10(14.9 \%)$ \\
\hline Neuroblastoma & $8(11.9 \%)$ \\
\hline Other & $38(56.7 \%)$ \\
\hline Age at cancer diagnosis (years) & $7.8 \pm 5.09$ \\
\hline Disease-free period (years) & $16.0 \pm 5.89$ \\
\hline \multicolumn{2}{|l|}{ Relapse history } \\
\hline No & 190 (95.0\%) \\
\hline Yes & $10(5.0 \%)$ \\
\hline \multicolumn{2}{|l|}{ Cancer treatment } \\
\hline Surgery & $65(32.5 \%)$ \\
\hline Radiotherapy (n=198) & $53(26.8 \%)$ \\
\hline Chemotherapy $(n=198)$ & $176(88.9 \%)$ \\
\hline \multicolumn{2}{|l|}{ Cancer treatment type $(n=196)$} \\
\hline Surgery only & $13(6.6 \%)$ \\
\hline Radiotherapy only & $3(1.5 \%)$ \\
\hline Chemotherapy only & $97(49.5 \%)$ \\
\hline Surgery and radiotherapy & $4(2.0 \%)$ \\
\hline Surgery and chemotherapy & $33(16.8 \%)$ \\
\hline Chemotherapy and radiotherapy & $31(15.8 \%)$ \\
\hline Surgery, chemotherapy, and radiotherapy & $15(7.7 \%)$ \\
\hline \multicolumn{2}{|l|}{ Education level $(n=196)$} \\
\hline Secondary or below & $66(33.7 \%)$ \\
\hline College/university or above & $130(66.3 \%)$ \\
\hline \multicolumn{2}{|l|}{ Occupation $(n=199)$} \\
\hline Employed/student/housewife & $185(93.0 \%)$ \\
\hline Unemployed & $14(7.0 \%)$ \\
\hline \multicolumn{2}{|l|}{ Smoking history } \\
\hline Non-smoker & $170(85.0 \%)$ \\
\hline Ex-smoker & $11(5.5 \%)$ \\
\hline Chronic smoker & 19 (9.5\%) \\
\hline \multicolumn{2}{|l|}{ Alcohol history } \\
\hline Non-drinker & $67(33.5 \%)$ \\
\hline Ex-drinker & $2(1.0 \%)$ \\
\hline Social drinker & $123(61.5 \%)$ \\
\hline Regular drinker & $8(4.0 \%)$ \\
\hline \multicolumn{2}{|l|}{ Marital status } \\
\hline Married & $31(15.5 \%)$ \\
\hline Single with current or previous relationship & $94(47.0 \%)$ \\
\hline Single and never in a relationship & $75(37.5 \%)$ \\
\hline \multicolumn{2}{|l|}{ Sexual history $(n=198)$} \\
\hline Yes & $80(40.4 \%)$ \\
\hline No & $118(59.6 \%)$ \\
\hline \multicolumn{2}{|l|}{ Reproductive history ( $n=197)$} \\
\hline Yes & $15(7.6 \%)$ \\
\hline No & $182(92.4 \%)$ \\
\hline
\end{tabular}

* Data are shown as No. (\%) or mean \pm standard deviation correlated with BIS score $(r=0.260, \mathrm{P}<0.001)$. Patients who had not undergone surgery $(4.9 \pm 4.09)$ had significantly lower BIS score than patients who had undergone surgery $(7.1 \pm 4.81, \mathrm{P}=0.002)$. Patients who had a history of haematological cancer $(4.9 \pm 4.11)$ also had significantly lower BIS score than patients who had a history of non-haematological cancer (7.1 \pm 4.77, $\mathrm{P}=0.002$ ) [online Supplementary Appendix 1]. Multiple regression analysis suggested that age at diagnosis $(\beta=0.22, \mathrm{P}<0.001)$ was associated with BIS score (online Supplementary Appendix 2).

The mean PHQ score was $4.80 \pm 4.27$. The numbers of patients who reported minimal depressive symptoms and major depression were 68 (34\%) and 24 (12\%), respectively (online Supplementary Appendix 3). No statistical significance was found across demographics variables (online Supplementary Appendices 1 and 2).

The General Health Questionnaire Short Form-12 analysis revealed that the overall PCS was $51.2 \pm 6.44$. Age at the time of this study $(r=-0.159$, $\mathrm{P}=0.025)$ and age at diagnosis $(r=-0.170, \mathrm{P}=0.017)$ were significantly negatively correlated with PCS. Patients who had undergone surgery without external effects $(51.9 \pm 5.20)$ had significantly higher mean PCS (ie, better physical health) than patients who had undergone surgery with external effects $(47.5 \pm$ 7.39, $\mathrm{P}=0.018$ ) [online Supplementary Appendix 1]. Multiple regression analysis showed that male sex $(\beta=2.04, P=0.024)$ was significantly associated with PCS, following adjustment for other variables (online Supplementary Appendix 2). In contrast, the overall MCS was $49.0 \pm 9.00$. There were no statistically significant relationships between MCS and any demographic variables (online Supplementary Appendices 1 and 2).

\section{Subgroup analysis based on sexual functioning scores}

Patients were divided into three groups based on their sexual functioning scores. Forty-eight (24.0\%) patients had experienced at least one sexual problem, $125(62.5 \%)$ patients reported that they never had any sexual problem and/or stated 'not applicable', and 27 (13.5\%) patients reported 'not applicable' for all items. Among women in this study, 19 (20.9\%) reported at least a small problem in at least one aspect of sexual function, 49 (53.8\%) reported 'not a problem' and/or 'not applicable' for all items, and 23 (25.3\%) reported 'not applicable' for all items; among men in this study, these numbers were 29 (26.6\%), 76 (69.7\%), and four (3.7\%), respectively (Table 4).

In the group with no sexual problems, more patients had haematological cancers $(n=89,71.2 \%$ vs $\mathrm{n}=24,50.0 \% ; \mathrm{P}=0.009)$, fewer patients underwent surgery $(n=34,27.2 \%$ vs $n=24,50.0 \% ; P=0.004)$, fewer patients underwent surgery with external effects $(\mathrm{n}=9,26.5 \%$ vs $\mathrm{n}=13,54.2 \% ; \mathrm{P}=0.032)$, and fewer 
TABLE 2. Sexual function (medical outcomes study sexual functioning score)*

\begin{tabular}{|c|c|c|c|c|}
\hline & No. of patients & \multicolumn{2}{|c|}{ Sexual functioning score } & $P$ value \\
\hline Overall & 200 & \multicolumn{2}{|c|}{$28.3 \pm 20.09$} & - \\
\hline \multicolumn{5}{|l|}{ Sex } \\
\hline Female & 91 & \multicolumn{2}{|c|}{$23.6 \pm 19.36$} & 0.002 \\
\hline Male & 109 & \multicolumn{2}{|c|}{$32.3 \pm 19.92$} & \\
\hline \multicolumn{5}{|l|}{ Cancer type } \\
\hline Non-haematological & 67 & \multicolumn{2}{|c|}{$31.0 \pm 20.28$} & 0.190 \\
\hline Haematological & 133 & \multicolumn{2}{|c|}{$27.0 \pm 19.94$} & \\
\hline \multicolumn{5}{|l|}{ Surgery } \\
\hline No & 135 & \multicolumn{2}{|c|}{$27.0 \pm 19.79$} & 0.170 \\
\hline Yes & 65 & \multicolumn{2}{|c|}{$31.2 \pm 20.57$} & \\
\hline \multicolumn{5}{|l|}{ External effect $(n=65)$} \\
\hline No & 42 & \multicolumn{2}{|c|}{$27.5 \pm 20.66$} & 0.054 \\
\hline Yes & 23 & \multicolumn{2}{|c|}{$37.8 \pm 19.08$} & \\
\hline \multicolumn{5}{|l|}{ Radiotherapy ( $n=198$ ) } \\
\hline No & 145 & \multicolumn{2}{|c|}{$26.9 \pm 18.36$} & 0.156 \\
\hline Yes & 53 & \multicolumn{2}{|c|}{$32.2 \pm 24.28$} & \\
\hline \multicolumn{5}{|l|}{ Chemotherapy $(n=198)$} \\
\hline No & 22 & \multicolumn{2}{|c|}{$27.3 \pm 18.05$} & 0.792 \\
\hline Yes & 176 & \multicolumn{2}{|c|}{$28.5 \pm 20.48$} & \\
\hline \multicolumn{5}{|l|}{ Relapse history } \\
\hline No & 190 & \multicolumn{2}{|c|}{$28.2 \pm 19.91$} & 0.570 \\
\hline Yes & 10 & \multicolumn{2}{|c|}{$31.9 \pm 24.20$} & \\
\hline \multicolumn{5}{|l|}{ Education level $(n=196)$} \\
\hline Secondary or below & 66 & \multicolumn{2}{|c|}{$28.3 \pm 17.41$} & 0.949 \\
\hline College/university or above & 130 & & \pm 21.34 & \\
\hline Occupation $(n=199)$ & & & & \\
\hline Unemployed & 14 & & \pm 19.24 & 0.900 \\
\hline Employed/student/housewife & 185 & & \pm 20.25 & \\
\hline Sexual history $(n=198)$ & & & & \\
\hline No & 118 & & \pm 20.07 & 0.008 \\
\hline Yes & 80 & & \pm 19.45 & \\
\hline Reproductive history $(\mathrm{n}=197)$ & & & & \\
\hline No & 182 & & \pm 20.33 & 0.355 \\
\hline Yes & 15 & & \pm 18.22 & \\
\hline Marital status & & & & \\
\hline Single and never in a relationship & 75 & & \pm 20.15 & 0.004 \\
\hline Single with current or previous relationship & 94 & & \pm 18.89 & \\
\hline Married & 31 & & \pm 20.63 & \\
\hline & $\begin{array}{l}\text { Correlation } \\
\text { coefficient }\end{array}$ & P value & $\begin{array}{l}\text { Linear regression } \\
\text { coefficient }\end{array}$ & $P$ value \\
\hline Age & 0.193 & 0.006 & 0.696 & 0.006 \\
\hline Age at diagnosis & 0.147 & 0.037 & 0.582 & 0.037 \\
\hline Disease-free period & 0.073 & 0.303 & 0.250 & 0.303 \\
\hline Body mass index & 0.049 & 0.490 & 0.273 & 0.490 \\
\hline
\end{tabular}

* Data are shown as mean \pm standard deviation, unless otherwise specified 
TABLE 3. Multiple regression analysis of medical outcomes study sexual functioning scale

\begin{tabular}{|c|c|c|c|c|c|c|}
\hline & \multicolumn{6}{|c|}{ Medical outcomes study sexual functioning scale } \\
\hline & \multicolumn{3}{|c|}{$\begin{array}{c}\text { Full model } \\
\text { (all demographics variables included) }\end{array}$} & \multicolumn{3}{|c|}{$\begin{array}{c}\text { Reduced model } \\
\text { (after model selection) }\end{array}$} \\
\hline & $\beta$ & P value & $\begin{array}{l}\text { 95\% Confidence } \\
\text { interval }\end{array}$ & $\beta$ & P value & $\begin{array}{l}95 \% \text { Confidence } \\
\text { interval }\end{array}$ \\
\hline (Constant) & 3.76 & 0.782 & -23.02 to 30.54 & 17.49 & $<0.001$ & 11.79 to 23.19 \\
\hline Sex (male) & 9.20 & 0.001 & 3.57 to 14.84 & 9.06 & 0.001 & 3.71 to 14.41 \\
\hline Age & 0.02 & 0.959 & -0.63 to 0.67 & & & \\
\hline Age at diagnosis & 0.30 & 0.310 & -0.28 to 0.89 & & & \\
\hline Haematological cancer & 4.69 & 0.643 & -15.22 to 24.59 & & & \\
\hline \multicolumn{7}{|l|}{ Surgery type } \\
\hline Non-external effect surgery & 8.05 & 0.451 & -12.98 to 29.07 & 2.15 & 0.525 & -4.51 to 8.81 \\
\hline External effect surgery & 13.41 & 0.200 & -7.15 to 33.98 & 9.49 & 0.029 & 0.99 to 17.99 \\
\hline Radiotherapy & 5.79 & 0.093 & -0.97 to 12.55 & & & \\
\hline Chemotherapy & 4.81 & 0.308 & -4.47 to 14.08 & & & \\
\hline Relapse history & 0.61 & 0.925 & -12.08 to 13.3 & & & \\
\hline Sexual history & 1.85 & 0.612 & -5.33 to 9.02 & & & \\
\hline Reproductive history & -3.78 & 0.582 & -17.27 to 9.72 & & & \\
\hline \multicolumn{7}{|l|}{ Marital status } \\
\hline Single with current or previous relationship & 3.79 & 0.275 & -3.04 to 10.61 & 4.75 & 0.109 & -1.06 to 10.57 \\
\hline Married & 13.95 & 0.038 & 0.79 to 27.1 & 13.83 & 0.001 & 5.75 to 21.91 \\
\hline
\end{tabular}

TABLE 4. Response to medical outcomes study sexual functioning scale

\begin{tabular}{|c|c|c|c|c|c|}
\hline & \multicolumn{3}{|c|}{ Sexual function } & \multirow[t]{2}{*}{ Total } & \multirow[t]{2}{*}{$P$ value } \\
\hline & $\begin{array}{l}\text { All answers = } \\
\text { 'Not applicable' }\end{array}$ & $\begin{array}{l}\text { All answers = } \\
\text { 'No problem' }\end{array}$ & $\begin{array}{l}\text { Sexual function } \\
\text { problem reported }\end{array}$ & & \\
\hline \multicolumn{6}{|l|}{ Sex } \\
\hline Female & 23 & 49 & 19 & 91 & $<0.001$ \\
\hline Male & 4 & 76 & 29 & 109 & \\
\hline Total & 27 & 125 & 48 & 200 & \\
\hline
\end{tabular}

patients were regular social drinkers $(n=2,1.6 \%$ vs $\mathrm{n}=6,12.5 \% ; \mathrm{P}=0.013)$. The group with no sexual problems also had statistically significantly higher PCS $(52.5 \pm 5.62$ vs $48.1 \pm 7.96, \mathrm{P}=0.003)$, higher Rosenberg self-esteem scale score ( $30.6 \pm 4.24$ vs 28.4 $\pm 4.06, \mathrm{P}=0.010)$, lower mean BIS score $(4.9 \pm 3.89 \mathrm{vs}$ $7.5 \pm 5.38, \mathrm{P}=0.008)$, and lower mean PHQ score (4.1 \pm 4.44 vs. $6.8 \pm 3.89, \mathrm{P}=0.001$ ) [Table 5]. However, in multivariable logistic regression analysis controlling for all potential confounders, no variables were statistically significant when comparing the group with no problems to the group with problems. After model selection, a history of surgery with external effects (odds ratio $[\mathrm{OR}]=6.09, \mathrm{P}=0.001$ ), PCS $(\mathrm{OR}=0.93, \mathrm{P}=0.010)$, and Rosenberg self-esteem scale score $(\mathrm{OR}=0.89, \mathrm{P}=0.013)$ were significantly related to the presence of sexual function problems (Table 6).

\section{Discussion}

\section{Summary}

In this study, approximately one-quarter of young Chinese cancer survivors in Hong Kong reported at least one sexual problem. Sexual problems were more common in men, in patients diagnosed with cancer at an older age, and in patients who were married or had a history of sexual experiences. Moreover, the presence of sexual problems was significantly associated with a history of surgery, as well as a history of surgery with external effects; patients with sexual problems generally had lower physical well-being scores, lower self-esteem scores, higher BIS scores, and an increased number of depressive symptoms. This new information can aid in understanding our patients' needs and in guiding the provision of necessary care. 
TABLE 5. Comparison between patients without sexual functioning problems (all responses to medical outcomes study sexual functioning scale = "no problem") and those with reported sexual functioning problems (any sexual functioning problem reported on medical outcomes study sexual functioning scale)*

\begin{tabular}{|c|c|c|c|}
\hline & $\begin{array}{c}\text { Patients without sexual } \\
\text { functioning problem }(n=125)\end{array}$ & $\begin{array}{c}\text { Patients with sexual } \\
\text { functioning problem }(n=48)\end{array}$ & $P$ value \\
\hline \multicolumn{4}{|l|}{ Sex } \\
\hline Female & $49(39.2 \%)$ & 19 (39.6\%) & 0.963 \\
\hline Male & 76 (60.8\%) & $29(60.4 \%)$ & \\
\hline \multicolumn{4}{|l|}{ Cancer type } \\
\hline Non-haematological & $36(28.8 \%)$ & $24(50.0 \%)$ & 0.009 \\
\hline Haematological & $89(71.2 \%)$ & $24(50.0 \%)$ & \\
\hline \multicolumn{4}{|l|}{ Surgery } \\
\hline No & $91(72.8 \%)$ & $24(50.0 \%)$ & 0.004 \\
\hline Yes & $34(27.2 \%)$ & $24(50.0 \%)$ & \\
\hline \multicolumn{4}{|l|}{ External effect $†$} \\
\hline No & $25(73.5 \%)$ & $11(45.8 \%)$ & 0.032 \\
\hline Yes & $9(26.5 \%)$ & $13(54.2 \%)$ & \\
\hline \multicolumn{4}{|l|}{ Radiotherapy $†$} \\
\hline No & $92(74.2 \%)$ & $32(68.1 \%)$ & 0.424 \\
\hline Yes & $32(25.8 \%)$ & $15(31.9 \%)$ & \\
\hline \multicolumn{4}{|l|}{ Chemotherapy $\dagger$} \\
\hline No & $14(11.3 \%)$ & $5(10.6 \%)$ & 0.904 \\
\hline Yes & $110(88.7 \%)$ & $42(89.4 \%)$ & \\
\hline \multicolumn{4}{|l|}{ Relapse history } \\
\hline No & $119(95.2 \%)$ & $45(93.8 \%)$ & 0.709 \\
\hline Yes & $6(4.8 \%)$ & $3(6.3 \%)$ & \\
\hline \multicolumn{4}{|l|}{ Education level† } \\
\hline Secondary or below & $41(33.3 \%)$ & $17(36.2 \%)$ & 0.727 \\
\hline College/university or above & $82(66.7 \%)$ & $30(63.8 \%)$ & \\
\hline \multicolumn{4}{|l|}{ Occupation† } \\
\hline Unemployed & $8(6.5 \%)$ & $4(8.3 \%)$ & 0.740 \\
\hline Employed/student/housewife & $116(93.5 \%)$ & $44(91.7 \%)$ & \\
\hline \multicolumn{4}{|l|}{ Sexual history $\dagger$} \\
\hline No & 73 (58.9\%) & $22(46.8 \%)$ & 0.156 \\
\hline Yes & $51(41.1 \%)$ & $25(53.2 \%)$ & \\
\hline \multicolumn{4}{|l|}{ Reproductive history $\dagger$} \\
\hline No & $112(91.1 \%)$ & $43(91.5 \%)$ & 1.000 \\
\hline Yes & $11(8.9 \%)$ & $4(8.5 \%)$ & \\
\hline \multicolumn{4}{|l|}{ Marital status } \\
\hline Single and never in a relationship & $42(33.6 \%)$ & $15(31.3 \%)$ & 0.140 \\
\hline Single with current or previous relationship & $65(52.0 \%)$ & $20(41.7 \%)$ & \\
\hline Married & $18(14.4 \%)$ & $13(27.1 \%)$ & \\
\hline \multicolumn{4}{|l|}{ Smoking history } \\
\hline Non-smoker & $105(84.0 \%)$ & $39(81.3 \%)$ & 0.439 \\
\hline Ex-smoker & $9(7.2 \%)$ & $2(4.2 \%)$ & \\
\hline Chronic smoker & $11(8.8 \%)$ & $7(14.6 \%)$ & \\
\hline \multicolumn{4}{|l|}{ Alcohol history } \\
\hline Non-drinker & $35(28.0 \%)$ & $15(31.3 \%)$ & 0.013 \\
\hline Ex-drinker & $1(0.8 \%)$ & $1(2.1 \%)$ & \\
\hline Social drinker & 87 (69.6\%) & $26(54.2 \%)$ & \\
\hline Regular drinker & $2(1.6 \%)$ & $6(12.5 \%)$ & \\
\hline Age (years) & $25.2 \pm 5.52$ & $27.0 \pm 5.82$ & 0.171 \\
\hline Age at diagnosis (years) & $7.6 \pm 4.92$ & $8.9 \pm 5.58$ & 0.281 \\
\hline Disease-free period (years) & $15.9 \pm 5.81$ & $16.6 \pm 6.08$ & 0.808 \\
\hline Body mass index $\left(\mathrm{kg} / \mathrm{m}^{2}\right)$ & $22.8 \pm 3.70$ & $21.7 \pm 3.40$ & 0.225 \\
\hline Physical component score & $52.5 \pm 5.62$ & $48.1 \pm 7.96$ & 0.003 \\
\hline Mental component score & $49.7 \pm 9.10$ & $47.1 \pm 8.66$ & 0.215 \\
\hline Rosenberg self-esteem scale & $30.6 \pm 4.24$ & $28.4 \pm 4.06$ & 0.010 \\
\hline Body image scale & $4.9 \pm 3.89$ & $7.5 \pm 5.38$ & 0.008 \\
\hline Patient Health Questionnaire & $4.1 \pm 4.44$ & $6.8 \pm 3.89$ & 0.001 \\
\hline
\end{tabular}

* Data are shown as No. (\%) or mean \pm standard deviation, unless otherwise specified

† Missing data are excluded from analysis 
TABLE 6. Multiple logistic regression analysis of medical outcomes study sexual functioning scores with other parameters

\begin{tabular}{|c|c|c|c|c|c|c|c|c|}
\hline \multirow[t]{2}{*}{$\begin{array}{l}\text { Ref group: No-problem } \\
\text { group }\end{array}$} & \multicolumn{4}{|c|}{$\begin{array}{l}\text { Full model (all demographics variables and study } \\
\text { measures included) }\end{array}$} & \multicolumn{4}{|c|}{ Reduced model (after model selection) } \\
\hline & B & $P$ value & Odds ratio & $\begin{array}{l}95 \% \mathrm{Cl} \text { for } \\
\text { Exp (B) }\end{array}$ & B & $P$ value & Odds ratio & $\begin{array}{l}95 \% \mathrm{Cl} \text { for } \\
\operatorname{Exp}(\mathrm{B})\end{array}$ \\
\hline Sex (Ref: Female) & 0.247 & 0.578 & 1.281 & $0.535-3.065$ & & & & \\
\hline Age & 0.025 & 0.616 & 1.026 & $0.929-1.132$ & & & & \\
\hline Age at diagnosis & 0.006 & 0.900 & 1.006 & $0.920-1.099$ & & & & \\
\hline $\begin{array}{l}\text { Cancer type (Ref: Non- } \\
\text { haematological) }\end{array}$ & 0.882 & 0.562 & 2.416 & $0.122-47.789$ & & & & \\
\hline Surgery type (Ref: No surgery) & & 0.105 & & & & 0.004 & & \\
\hline Non-external effect surgery & 1.880 & 0.243 & 6.552 & $0.279-153.727$ & 0.740 & 0.106 & 2.095 & $0.853-5.144$ \\
\hline External effect surgery & 2.787 & 0.071 & 16.226 & $0.789-333.681$ & 1.807 & 0.001 & 6.090 & $2.005-18.498$ \\
\hline Radiotherapy (Ref: No) & 0.125 & 0.800 & 1.133 & $0.430-2.983$ & & & & \\
\hline Chemotherapy (Ref: No) & 0.595 & 0.401 & 1.813 & $0.452-7.271$ & & & & \\
\hline Relapse history (Ref: No) & -0.079 & 0.937 & 0.924 & $0.129-6.626$ & & & & \\
\hline Sexual history (Ref: No) & 0.490 & 0.389 & 1.633 & $0.535-4.981$ & & & & \\
\hline Reproductive history (Ref: No) & -1.047 & 0.264 & 0.351 & $0.056-2.204$ & & & & \\
\hline $\begin{array}{l}\text { Marital status (Ref: Single and } \\
\text { never in a relationship) }\end{array}$ & & 0.419 & & & & & & \\
\hline $\begin{array}{l}\text { Single with current or } \\
\text { previous relationship }\end{array}$ & -0.575 & 0.549 & 0.563 & $0.086-3.693$ & & & & \\
\hline Married & -0.913 & 0.239 & 0.401 & $0.088-1.836$ & & & & \\
\hline Physical component score & -0.072 & 0.060 & 0.930 & $0.863-1.003$ & -0.075 & 0.010 & 0.928 & $0.876-0.982$ \\
\hline Mental component score & -0.037 & 0.238 & 0.963 & $0.905-1.025$ & & & & \\
\hline Rosenberg self-esteem scale & -0.077 & 0.200 & 0.926 & $0.824-1.041$ & -0.120 & 0.013 & 0.887 & $0.807-0.975$ \\
\hline Body image scale & -0.006 & 0.927 & 0.994 & $0.880-1.123$ & & & & \\
\hline Patient Health Questionnaire & 0.017 & 0.784 & 1.018 & $0.898-1.152$ & & & & \\
\hline Constant & 4.429 & 0.267 & 83.835 & & 5.979 & 0.002 & 395.099 & \\
\hline
\end{tabular}

Abbreviation: $95 \% \mathrm{Cl}=$ confidence interval

\section{Sex-related differences in sexual function outcomes}

In a similar study in the United States, ${ }^{4}$ involving 599 cancer survivors aged 18 to 39 years, $52 \%$ of female and $32 \%$ of male respondents reported at least 'a small problem' in one or more areas of sexual functioning. Overall, $42.7 \%$ of the patients in that study reported at least one problematic symptom; the overall sexual functioning score (indicative of more problems) was higher in women (21.6) than in men (10.6). Interestingly, the findings in our study contrasted with those of the prior study. Approximately onequarter of survivors (24\% overall, $26.6 \%$ of men, $20.9 \%$ of women) had at least one sexual problem. Furthermore, the overall sexual functioning scores for male and female survivors were 32.3 and 23.6, respectively. Therefore, fewer cancer survivors may experience sexual problems in Hong Kong. However, the problems experienced by these survivors may be more severe, as reflected by the higher sexual functioning score.

In our study, men had higher sexual functioning scores (ie, more sexual problems) than women. However, compared with men in the study (3.7\%), many more women (25.3\%) reported 'not applicable' $(\mathrm{P}<0.001)$. An overall lower score among women may not necessarily mean that they experienced fewer sexual problems; it might indicate that they were less sexually active. By excluding responses of 'not applicable' from the overall sexual functioning scale assessment, we found no significant sex-related difference in sexual functioning score $(\mathrm{P}=0.499)$. The mean scores for women and men were $31.62 \pm 15.72$ and $33.51 \pm 19.25$, respectively. Regarding patients with responses of 'not applicable' in the overall sexual functioning scale, $85.2 \%$ did not have sexual experience. Furthermore, women in the present study may have been less sexually active than men. A larger proportion of female survivors might only have sexual intercourse after marriage and thus be unaware of sexual problems prior to that point. Therefore, long-term assessment of sexual function is important for identifying sexual problems in cancer survivors, especially women.

\section{Sex-related differences in specific sexual problems}

Overall, the most common sexual problems reported 
were difficulties in relaxing and enjoying sex (19.5\%) and difficulties in achieving an erection or orgasm (18.5\%). Comparatively fewer survivors reported lack of sexual interest (13.0\%) and problems in becoming sexually aroused (13.5\%). Frederick et $\mathrm{al}^{16}$ performed a semi-structured interview study in a paediatric oncology and survivorship clinic, involving 22 childhood cancer survivors aged 18 to 39 who reported two or more sexual problems. The most commonly reported sexual problems were also difficulties in relaxing and enjoying sex $(n=19,86 \%)$ and difficulties in achieving an erection or orgasm $(n=18,82 \%)$, as in the present study. Frederick et $\mathrm{al}^{16}$ also reported that for each of the sexual function items, the proportion of women who reported problems (34.1\%-39.5\%) was greater than the proportion of men who reported problems (15.3\%-20.4\%). However, our study showed similar proportions of women and men experiencing problems in becoming sexually aroused (women: 13.2\%, men: 13.8\%) and in achieving an erection or orgasm (women: 18.7\%, men: 18.3\%). A greater proportion of men reported a lack of sexual interest (women: 8.8\%, men: 16.5\%) and an inability to relax or enjoy sex (women: $17.6 \%$, men: $21.1 \%$ ). The sexual problems experienced by cancer survivors seemed to differ between sexes. In Chinese culture, men play a more dominant role in a relationship, and typically initiate sexual activity. ${ }^{17}$ This might be why more men reported problems regarding sexual desire, including sexual interest, relaxation, and enjoyment. Because Asian women are more passive in terms of sexual activity, they might not view reduced sexual interest as a problem. ${ }^{18}$ Instead, they might be more concerned with an inability to achieve orgasm during sex.

The authors of previous studies proposed that greater numbers of female survivors reported sexual problems because they were more likely to experience cancer-related physical changes and psychological distress. ${ }^{4,19}$ However, our study showed no significant sex-related differences in physical health $(\mathrm{P}=0.072)$, mental health $(\mathrm{P}=0.354)$, self-esteem $(\mathrm{P}=0.184)$, body image $(\mathrm{P}=0.057)$, or depressive symptoms $(\mathrm{P}=0.349)$. This implies that the cancer survivors in our study did not experience sex-specific effects of their childhood cancer experience on their quality of life.

\section{Implications for patient treatment}

It is well-known that treatments for cancer may cause adverse effects on sexual function. Both Kenney et $\mathrm{al}^{20}$ and Van Dorp et $\mathrm{al}^{21}$ reviewed the literature regarding reproductive health of male and female survivors. They noted that alkylating agent chemotherapy and gonadal irradiation carried doserelated risks of primary gonadal dysfunction, which affected both sexual function and fertility. Chow et $\mathrm{a}^{22}$ also stated that surgery might involve longterm consequences, disfiguration with psychosocial impact, and delayed complications. Our study found that a larger proportion of survivors who had undergone surgery, especially surgery with external effects, reported problems involving sexual function, whereas survivors who had undergone radiation or chemotherapy showed no significant difference between the proportions of survivors who reported the presence or absence of problems involving sexual function.

Adolescence and young adulthood are the points in life when people focus intensely on their own bodies and can experience dissatisfaction with their bodies and physical appearances. ${ }^{23}$ Any alterations in physical appearance may affect their self-perceptions. Indeed, in a study involving focus groups and questionnaire surveys among survivors aged 15 to 29 years and matched controls to investigate body image and sexual health among adolescents and young adult cancer survivors, Olsson et $\mathrm{a}^{24}$ found that survivors perceived themselves to be less sexually attractive due to scars on their bodies and were less satisfied with their sexual function, compared with their matched controls.

With the progression of surgical techniques, such as the introduction of minimally invasive surgery, we presume that the impacts of scarring and physical disfiguration may be minimised. Until this change occurs, healthcare professionals should provide information regarding the potential adverse effects of treatments on the reproductive system and sexual function, as well as counselling to the survivors; importantly, survivors interviewed in previous studies indicated they had unmet needs for information, support, and counselling. ${ }^{20}$

\section{Limitations}

There were some limitations in our study. Because we did not include a control arm, we could not assess whether there were any differences between our patients and similar age-matched young adults in terms of the measured parameters. Therefore, we plan to perform a follow-up study that involves the application of the assessments in these questionnaires to similarly aged individuals in the general population to confirm our findings. Another limitation of this study was that it was performed in a single centre and the findings may be biased due to the specific patient population involved. However, this is one of the main children's cancer centres in Hong Kong, and is therefore a major referral centre that receives patients from various regions of Hong Kong; combined with the moderate sample size, we consider this to provide a good representation of adult survivors of childhood cancer in Hong Kong.

\section{Conclusion}

In this cross-sectional study of 200 young Chinese cancer survivors, approximately one-quarter of 
the patients reported at least one sexual problem. A history of sexual problems was significantly associated with a history of surgery, as well as a history of surgery with external effects. Compared with patients without sexual problems, those with sexual problems generally had lower physical wellbeing scores, lower self-esteem scores, higher body image distress scores, and an increased number of depressive symptoms. Given the findings in this study, aspects of life beyond disease condition and physical function should be considered in adult survivors of childhood cancers. Moreover, appropriate referral and intervention should be initiated for these patients when necessary.

\section{Author contributions}

All authors had full access to the data, contributed to the study, approved the final version for publication, and take responsibility for its accuracy and integrity.

Concept or design: CF Ng, AWK Leung.

Acquisition of data: BSY Lau, CF Ng, AWK Leung.

Analysis or interpretation of data: CYL Hong, BSY Lau, CF Ng.

Drafting of the article: CYL Hong, BSY Lau, CF Ng.

Critical revision for important intellectual content: All authors.

\section{Conflicts of interest}

As editors of the journal, CF $\mathrm{Ng}$ and JYC Teoh were not involved in the peer review process. Other authors have no conflicts of interest to disclose.

\section{Funding/support}

The project was supported by Hong Kong Children Cancer Fund.

\section{Ethics approval}

Approvals (CRE-2014.674) from The Joint Chinese University of Hong Kong-New Territories East Cluster Clinical Research Ethics Committee were obtained.

\section{References}

1. World Health Organization. Sexual health-a new focus for WHO. Prog Reprod Health Res 2004;67:1-8.

2. Gan HW, Spoudeas HA. Long-term follow-up of survivors of childhood cancer (SIGN Clinical Guideline 132). Arch Dis Child Educ Pract Ed 2014;99:138-43.

3. Jacobs LA, Pucci DA. Adult survivors of childhood cancer: the medical and psychosocial late effects of cancer treatment and the impact on sexual and reproductive health. J Sex Med 2013;10 Suppl 1:120-6.

4. Zebrack BJ, Foley S, Wittmann D, Leonard M. Sexual functioning in young adult survivors of childhood cancer. Psychooncology 2010;19:814-22.

5. Sundberg KK, Lampic C, Arvidson J, Helström L, Wettergren L. Sexual function and experience among long-term survivors of childhood cancer. Eur J Cancer 2011;47:397-403.

6. Bober SL, Zhou ES, Chen B, Manley PE, Kenney LB, Recklitis CJ. Sexual function in childhood cancer survivors: a report from project REACH. J Sex Med 2013;10:2084-
93.

7. Sherbourne C. Social functioning: sexual problems measures. In: Stewart AL, Ware JE, editors. Measuring Functioning and Well-being: the Medical Outcomes Study Approach. Durham (NC), United States: Duke University Press; 1992: 194-204.

8. Ware J Jr, Kosinski M, Keller SD. A 12-item short-form health survey: construction of scales and preliminary tests of reliability and validity. Med Care 1996;34:220-33.

9. Lam CL, Tse EY, Gandek B. Is the standard SF-12 health survey valid and equivalent for a Chinese population? Qual Life Res 2005;14:539-47.

10. Rosenberg M. Society and the Adolescent Self-image. Princeton [NJ], United States: Princeton University Press; 1965.

11. Li HC, Chung OK, Ho KY, Chiu SY, Lopez V. A descriptive study of the psychosocial well-being and quality of life of childhood cancer survivors in Hong Kong. Cancer Nurs 2012;35:447-55.

12. Hopwood P, Fletcher I, Lee A, Al Ghazal S. A body image scale for use with cancer patients. Eur J Cancer 2001;37:189-97.

13. Lin MS. An investigation on the body image, medication adherence and quality of life in patients with systemic lupus erythematosus after prednisolone treatment [thesis]. China Medical University, Taichung City, Taiwan; 2010.

14. Kroenke K, Spitzer RL, Williams JB. The PHQ-9: validity of a brief depression severity measure. J Gen Intern Med 2001;16:606-13.

15. Yu X, Tam WW, Wong PT, Lam TH, Stewart SM. The Patient Health Questionnaire-9 for measuring depressive symptoms among the general population in Hong Kong. Compr Psychiatry 2012;53:95-102.

16. Frederick NN, Recklitis CJ, Blackmon JE, Bober S. Sexual dysfunction in young adult survivors of childhood cancer. Pediatr Blood Cancer 2016;63:1622-8.

17. Lo SS, Kok WM. Sexual behavior and symptoms among reproductive age Chinese women in Hong Kong. J Sex Med 2014;11:1749-56.

18. Atallah S, Johnson-Agbakwu C, Rosenbaum $\mathrm{T}$, et al. Ethical and sociocultural aspects of sexual function and dysfunction in both sexes. J Sex Med 2016;13:591-606.

19. The Family Planning Association of Hong Kong. Report on youth sexuality study 2016. 2017. Available from: https:// www.famplan.org.hk/en/media-centre/press-releases/ detail/fpahk-report-on-youth-sexuality-study. Accessed 9 Jul 2018.

20. Kenney LB, Antal Z, Ginsberg JP, et al. Improving male reproductive health after childhood, adolescent, and young adult cancer: progress and future directions for survivorship research. J Clin Oncol 2018;36:2160-8.

21. van Dorp W, Haupt R, Anderson RA, et al. Reproductive function and outcomes in female survivors of childhood, adolescent, and young adult cancer: a review. J Clin Oncol 2018;36:2169-80.

22. Chow EJ, Antal Z, Constine LS, et al. New agents, emerging late effects, and the development of precision survivorship. J Clin Oncol 2018;36:2231-40.

23. Cash T. Encyclopedia of Body Image and Human Appearance. Oxford, United Kingdom: Elsevier Science; 2012.

24. Olsson M. Adolescent and Young Adult Cancer SurvivorsBody Image and Sexual Health. Gothenburg, Sweden: University of Gothenburg; 2018. 